\title{
Nutzung eines Vorrichtungsbaukastens für die Bearbeitung komplexer Aufgabenstellungen in Lehre und Praxis
}

\author{
Norbert Miersch
}

Zusammenfassung

Das Thema beschäftigt sich mit dem Einsatz von Baukastenvorrichtungen in Lehre und Praxis. Es werden Begriffe zum Vorrichtungsbau erläutert. Sonder- und Baukastenvorrichtung werden miteinander verglichen. Es wurde eine allgemeine Vorgehensweise entwickelt (Algorithmus). Der Ablauf für Planung und Montage einer Baukastenvorrichtung wird an einem praktischen Beispiel demonstriert. Es werden Vorteile bei der Nutzung eines 3D-CAD-Systems aufgezeigt. Der Artikel zeigt auf, dass die Vorrichtungsproblematik komplex ist. Es wird technologisches und konstruktives Wissen benötigt. Ein fachübergreifendes Arbeiten ist erforderlich. Es können zukünftig Aufgabenstellungen in Lehre und Praxis bearbeitet werden.
Abstract

The subject deals with the use of modular devices in teaching and practice. There are concepts introduced to the fixture. Special and modular device can be compared. We developed a general approach (algorithmus). The process for planning and installation of a modular device is demonstrated by a practical example. It identifies benefits of using a 3D CAD system. The article shows that the device problem is complex and requires technological and constructive knowledge. An inter-disciplinary work is required. In future problems in teaching and practice may be processed.

\section{Einleitung}

Im Studiengang Ingenieurwesen/Maschinenbau der TH Wildau (FH) wurde für die Lehre sowie die Bearbeitung von Aufgabenstellungen aus der Industrie ein Vorrichtungsbaukasten der Firma »Hohenstein Vorrichtungsbau und Spannsysteme GmbH« mit Nutsystem (T-Nut nach DIN 650) angeschafft. Solide Erfahrungen zum Vorrichtungsbau liegen durch die Bearbeitung verschiedener Aufgabenstellungen aus der Praxis im Lehrgebiet Vorrichtungskonstruktion vor (Miersch et al. 2003). Hier wurden bisher Sondervorrichtungen konstruiert.

Die Arbeit mit einem Vorrichtungsbaukasten setzt eine von den Sondervorrichtungen abweichende Vorgehensweise voraus. Mit einem Baukasten können unterschiedliche Vorrichtungen montiert werden.

In der Lehre beschränkt sich der Ablauf bei der Bearbeitung von Aufgabenstellungen nun nicht mehr wie bei Sondervorrichtungen auf die Konstruktions- und Planungsphase, sondern die theoretische Lösung wird praktisch umgesetzt und überprüft. Es entsteht eine montierte Vorrichtung.

Weiterhin ermöglicht der Vorrichtungsbaukasten die Bearbeitung von Aufgabenstellungen aus der industriellen Praxis. Solche Vorrichtungen werden in der Produktion (Null- und Kleinserienfertigung) oder im Versuchs- oder Prototypenbau bei der Entwicklung von neuen Produkten benötigt. Das Baukastensystem kann also auch Anwendung in angewandter Forschung und Entwicklung finden.

Der Vorrichtungsbaukasten mit dazugehörigen Montagewerkzeugen, Hilfsmittel zur Lagerhaltung und Montage, Rechner mit dem 3D-CAD-System SolidWorks und einer durch Studenten programmierten Software für die Vorrichtungsteileverwaltung wurden im Labor für Maschinenberechnung schrittweise integriert. In Teams können dann spezielle Aufgabenstellungen bearbeitet werden.

\section{Begriffsbestimmungen und Eingrenzungen}

Der Vorrichtungsbau ist ein Teilgebiet des Werkzeugbaues (Dolmetsch et al. 2007). Vorrichtungen werden im Maschinenbau insbesondere in den Branchen Werk- 
zeugmaschinenbau, Stahlbau, Kranbau, Fahrzeugbau sowie Luft- und Raumfahrttechnik in Herstellungs-, Montage-, Prüf- und Reparaturprozessen eingesetzt.

Betrachtet man nur den Herstellungsprozess, gibt es zugeordnet zu den Verfahrenshauptgruppen nach DIN 8580, verschiedene Arten von Vorrichtungen. wie beispielsweise Bohr-, Dreh-, Fräs-, Klebe-, Schweiß-, Schrumpf-, Beschichtungs- und Wärmebehandlungsvorrichtungen.

Für die nachfolgenden Ausführungen soll sich die Verwendung von Vorrichtungen auf die mechanische Bearbeitung von Einzelteilen beziehen. Nach DIN 6300 und (Perovic, 1999) sind Vorrichtungen Fertigungsmittel, die zu bearbeitende Werkstücke in eine bestimmte Lage zum bearbeitenden Werkzeug bringen und während der Bearbeitung in dieser Lage halten. Eine Vorrichtung muss zwei Grundfunktionen erfüllen:

1. Das Bestimmen: Entziehen aller erforderlichen Freiheitsgrade ${ }^{1}$ für eine reproduzierbare Lagesicherung.

2. Das Spannen: Halten des Werkstückes in der reproduzierbaren Lage während der Bearbeitung.

Neben den zwei Grundfunktionen gibt es nach (VDIGesellschaft 1992) Ergänzungsfunktionen. Dazu gehört beispielsweise das »Stützen«. Stützelemente schaffen eine zusätzliche Vorrichtungskontaktfläche.

Um die besondere Vorgehensweise bei der Konstruktion bzw. Montage von Baukastenvorrichtungen gegenüber Sondervorrichtungen herauszustellen, werden im folgenden Gliederungspunkt beide Vorrichtungen miteinander verglichen.

\section{Vergleich von Sonder- und Baukasten- vorrichtungen}

Baukastenvorrichtungen werden hauptsächlich in der Einzel- und Kleinserienfertigung, in der Produktforschung, oder für den Anlauf einer Serie eingesetzt. In der folgenden Tabelle 1 wird eine qualitative Bewertung beider Vorrichtungsarten vorgenommen.

Der Ablauf zur Bereitstellung beider Vorrichtungsarten unterscheidet sich im Wesentlichen nach dem Bestimmen und Spannen. Er wird in der Abb. 1 gegenübergestellt.

Die Nutzung eines 3D-CAD-Systems kann für eine virtuelle Vorrichtungsmontage genutzt werden. Dadurch verkürzen sich die Bereitstellungsfristen der Vorrichtung. Bei vorhandenen 3D-CAD-Daten des Baukastens und des Werkstückes kann die Vorrichtungsmontage,

\begin{tabular}{|l|l|l|}
\hline Kriterien & $\begin{array}{l}\text { Art der Vorrichtung } \\
\text { Sonder }\end{array}$ & Baukasten \\
\hline Flexibilität & gering & hoch \\
\hline Genauigkeit & sehr hoch möglich & hoch \\
\hline Zeit bis zur Verfügbarkeit & lang & kurz \\
\hline Steifigkeit & hoch & gering \\
\hline Systemkosten & niedrig & hoch \\
\hline für Serienfertigung & geeignet & weniger geeignet \\
\hline
\end{tabular}

Tab. 1 : Vergleich von Sonder- und Baukastenvorrichtung

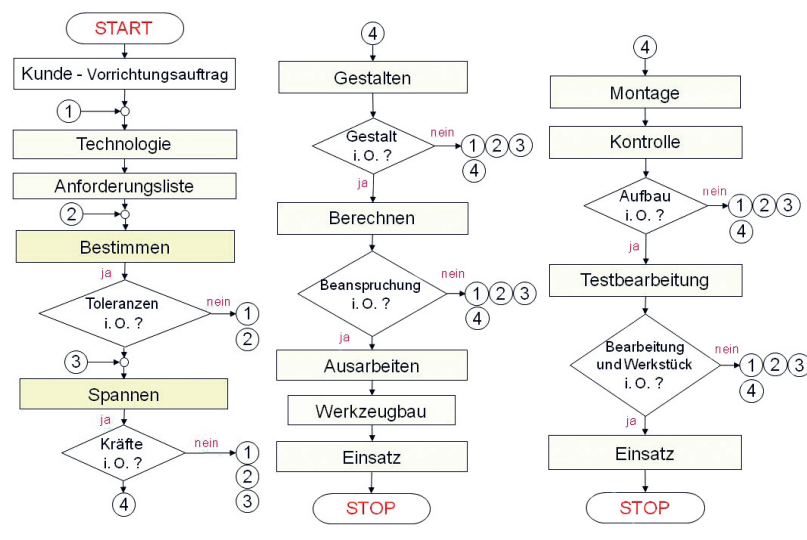

a) Sondervorrichtung b) Baukastenvorrichtung

Abb. 1: Ablauf der Konstruktion von Sonder- bzw. Montage von Baukastenvorrichtungen; a) Sondervorrichtung; b) Baukastenvorrichtung nach dem Spannen

die NC-Programmierung und NC-Simulation während der Lieferfrist des unbearbeiteten Werkstückes (Rohteiles) erfolgen.

Im Weiteren wird anhand eines repräsentativen Werkstückes aus der Praxis der komplexe Ablauf für die Planung und Montage einer Baukastenvorrichtung verdeutlicht.

\section{Planung und Montage einer Baukasten- vorrichtung}

Das repräsentative Werkstück wird im Folgenden als »Trägerplatte« bezeichnet. Die Trägerplatte soll in einer Aufspannung bearbeitet werden (siehe Abb. 2).

Beispielhaft wird der Ablauf bis zur Montage der Vorrichtung für die Bearbeitung der ersten Aufspannung entsprechend Abb. 1b) betrachtet.

Abb 2: Repräsentatives Werkstück

\section{Vorrichtungsauftrag}

Der Bedarf einer Vorrichtung entsteht bei der Erarbeitung der Technologie für das Werkstück ausgehend vom Rohteil. 


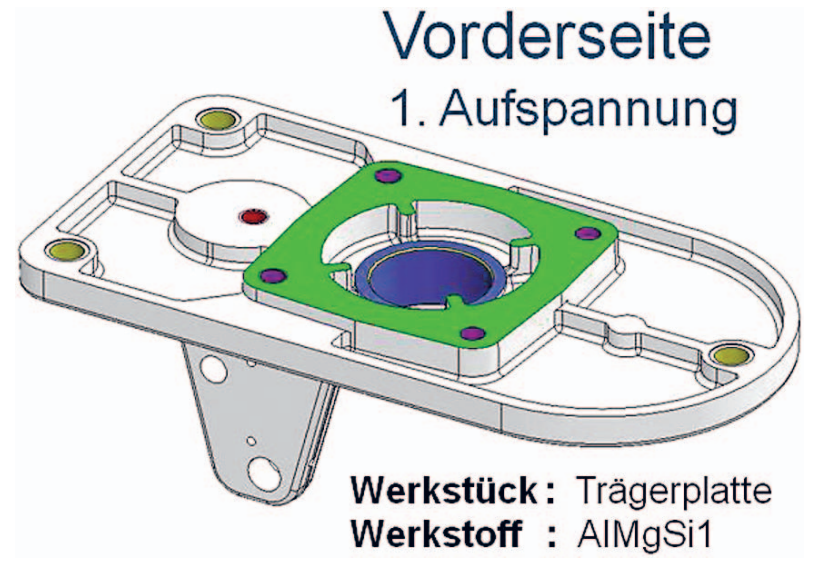

Technologie

Die Technologie berücksichtigt neben der Arbeitsvorgangsfolge wenn erforderlich auch eine Arbeitsstufenfolge. Die Arbeitsstufenfolge ist eine detaillierte Beschreibung des Arbeitsvorganges. Dazu gehören Informationen zum Fertigungsverfahren (Werkzeugmaschine), den technologischen Arbeitswerten sowie den Bearbeitungszeiten (Haupt- und Nebenzeiten). Formelemente wie Bohrungen, Fasen oder Flächen werden unter Berücksichtigung der Maß-, Form-, Lageund Oberflächentoleranzen in einer oder mehreren Aufspannungen auf einer Werkzeugmaschine mit verschiedenen Werkzeugen durchgeführt.

Beispielhaft wurde für die erste Aufspannung der Trägerplatte das Bearbeitungszentrum VC 560 (Standort Labor 14-A0.09) ausgewählt. Die nachfolgende Abb. 3 zeigt in der Reihenfolge der Positionsnummern ausgewählte Arbeitsstufen mit konkreten technologischen Arbeitswerten.

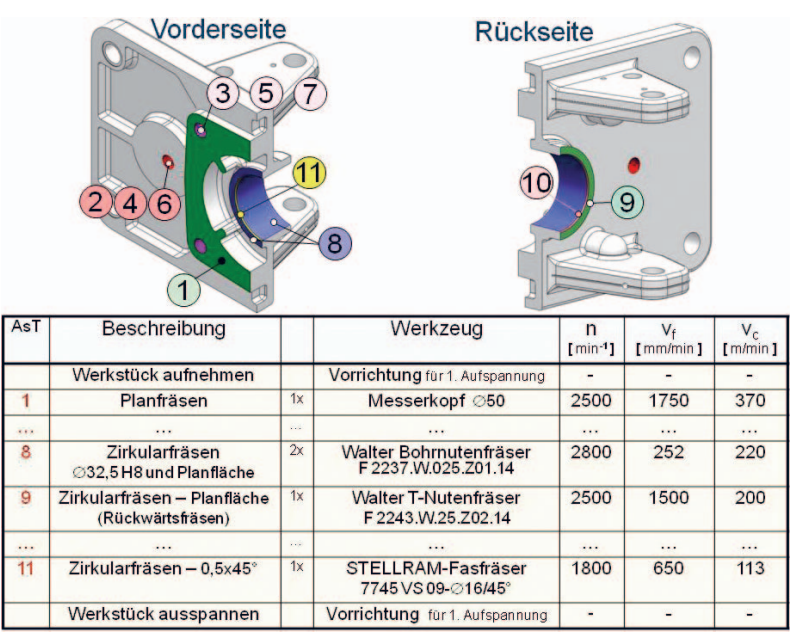

Abb. 3: Arbeitsstufenfolge der ersten Aufspannung für die Trägerplatte am BAZ VC 560
Für das ausgewählte BAZ VC 560 wurde in Arbeitsstufe 8 (Auftreten der größten Schnittkraft) nach Abb. 3 eine Schnittkraft von $785 \mathrm{~N}$ bei einem Leistungsbedarf von 3,2 kW berechnet. Dieser Leistungsbedarf liegt unter der Antriebsleistung des BAZ VC 560 mit 7,5 kW im Dauerbetrieb. Im Ergebnis der Berechnung kann die Werkzeugmaschine für die geplante Bearbeitung verwendet werden.

\section{Anforderungsliste}

Eine zweckmäßige Vorgehensweise in der Vorrichtungskonstruktion und -planung stellt die Nutzung einer Anforderungsliste dar. Diese wird vorzugsweise in der Produktkonstruktion verwendet. Die Anforderungsliste dient zur Klärung und genauen Festlegung der Aufgabe. Sie ist eine systematisch erarbeitete Zusammenstellung aller Daten und Informationen für den Vorrichtungskonstrukteur bzw. -monteur (Conrad 2010). In Tabelle 2 werden auszugsweise die wichtigsten Daten, die für die Montage der Baukastenvorrichtung der Trägerplatte erforderlich sind, zusammengestellt.

\begin{tabular}{|l|l|l|l|l|l|l}
\hline F/W & Beschreibung \\
\hline 1 & Werkstück/Vorrichtung \\
\hline F & drei Bohrungen $\varnothing 9,3 \mathrm{~mm}$ zum Bestimmen vorgefertigt \\
\hline W & keine Zukaufteile, Elemente des Baukastens nutzen \\
\hline 2 & Bestimmen \\
\hline F & Bestimmen in 2 Bohrungen, 6 Freiheitsgrade binden \\
\hline F & Toleranz der Vorrichtung $=1 / 3$ der Toleranz des Werkstückes \\
\hline 3 & Spannen \\
\hline F & mit Muskelkraft spannen $\mathrm{F}_{\mathrm{H}}=75 \mathrm{~N}$ \\
\hline $\mathrm{F}$ & Spannen gegen Auflagebolzen \\
\hline 4 & Bearbeitung \\
\hline F & Vorrichtung für die »erste Aufspannung « \\
\hline F & Werkzeugmaschine BAZ VC 560 \\
\hline
\end{tabular}

Tab. 2: Auszug der Anforderungsliste für die Baukastenvorrichtung der Trägerplatte; Legende: F-Forderung, W-Wunsch

\section{Bestimmen}

Um eine reproduzierbare Lage des Werkstückes in der Vorrichtung zu gewährleisten, werden Bestimmelemente genutzt. In Abb. 4 sind in einer Übersicht grundlegende Bestimmelemente mit der Anzahl der gebundenen Freiheitsgrade systematisiert. Man unterscheidet Bestimmelemente zum Bestimmen in der Außen- und Innenkontur des Werkstückes. Beispielsweise werden durch das Bestimmen mit einem vollen langen Bolzen 


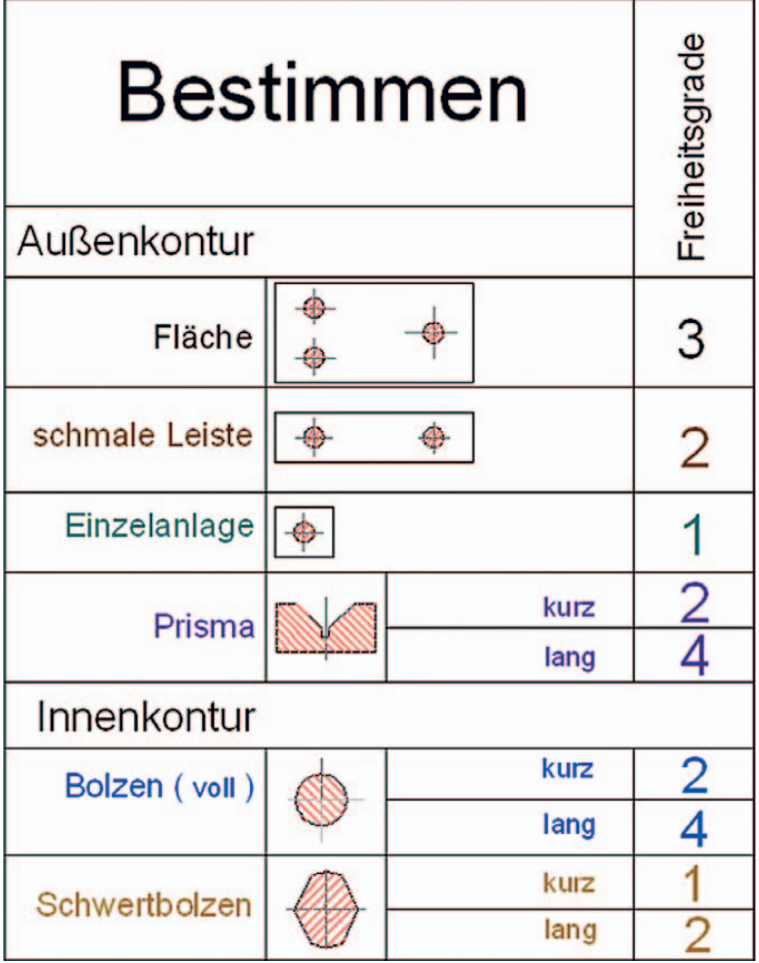

Abb. 4: Systematik der Hauptbestimmelemente

vier Freiheitsgrade gebunden (entzogen). Wird auf einer zylindrischen Außenkontur in einem langen Prisma bestimmt, werden auch hier vier Freiheitsgrade entzogen.

Die Bestimmelemente des Vorrichtungsbaukastens lehnen sich grundsätzlich an diese Systematik an. Da die Werkstücktoleranzen während der Fertigung bei reproduzierbarer Lage einzuhalten sind, gibt es Regeln. Im Folgenden sind die wichtigsten Regeln zum Bestimmen aufgeführt. Nähere Erläuterungen zu den Regeln sind in entsprechender Literatur (Trummler et al. 1994; Matuszewski 1986) gegeben.

Regel 1: Überbestimmen ist zu vermeiden!

Regel 2: Bringe Bestimm- und Bezugsebene zur Deckung!

Regel 3: Grenze die Vorrichtungstoleranz auf $1 / 3$ bis 1/5 der Werkstücktoleranz ein!

Regel 4: Führe den vollen Bolzen länger aus als den Schwertbolzen! (Für das Bestimmen in zwei Bohrungen.)

Für die Trägerplatte (Regel 2 konnte nicht für alle Maße eingehalten werden) waren Toleranzuntersuchungen erforderlich. In der folgenden Abb. 5 sind die Elemente des Vorrichtungsbaukastens für das Bestimmen der Trägerplatte in der 1. Aufspannung dargestellt (Trägerplatte schwach gezeichnet). Insgesamt wurden der Trägerplatte in dieser Aufspannung sechs Freiheitsgrade entzogen.

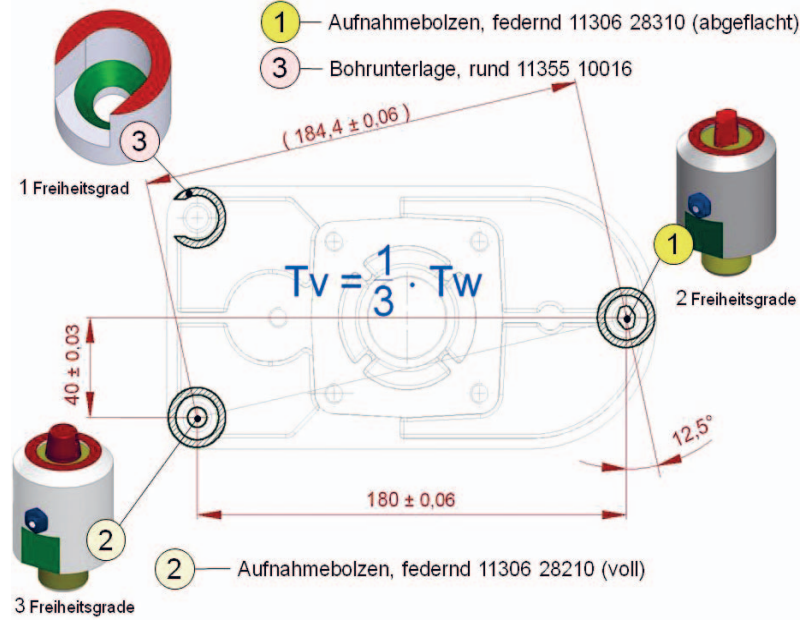

Abb. 5: Bestimmelemente der Trägerplatte in der 1. Aufspannung

Spannen

Bei Nutzung eines Vorrichtungsbaukastens werden in der Regel mechanische Spannelemente wie Spanneisen, Niederzugspanner, Spannschrauben, Spannexzenter und Spannspiralen eingesetzt. Im Allgemeinen werden in der Praxis keine Spannkräfte berechnet. Die meisten Spannelemente werden aufgrund von Erfahrungen eingesetzt und gehandhabt. Gegebenenfalls werden die Schnittdaten $\left(n, v_{f}, v_{c}\right)$ an der Werkzeugmaschine angepasst.

Um das Spannen reproduzierbar und fehlerfrei zu realisieren, wird empfohlen, folgende Regeln zu beachten, die wiederum in entsprechender Literatur (Trummler et al., 1994; Matuszewski, 1986) kommentiert werden.

Regel 1: Berechne Ersatzkraft (korrigierte Schnittkraft) und stelle Kräfteplan auf!

Regel 2: Berücksichtige Handkraft bei der Kraftübertragung!

Regel 3: Vermeide die Verspannung (offset) des Werkstückes!

Regel 4: Vermeide zu große Flächenpressungen am Werkstück!

Bezogen auf die Trägerplatte ergibt sich nach Abb. 6 folgender Kräfteplan. Zur Auswahl zweckmäßiger Spannelemente ist es ratsam, entsprechende Berechnungen zu den Spannelementen durchzuführen (z. B. Schraubenberechnungen).

\section{Montage}

Wie oben bereits erwähnt ist es zweckmäßig, auch in der industriellen Praxis (VDI-Gesellschaft 1992), die Vorrichtung in einem 3D-CAD-System zu montieren (Baugruppe). Der Lieferant der Baukastenvorrichtung (in diesem Fall die Firma Hohenstein Vorrichtungsbau und 


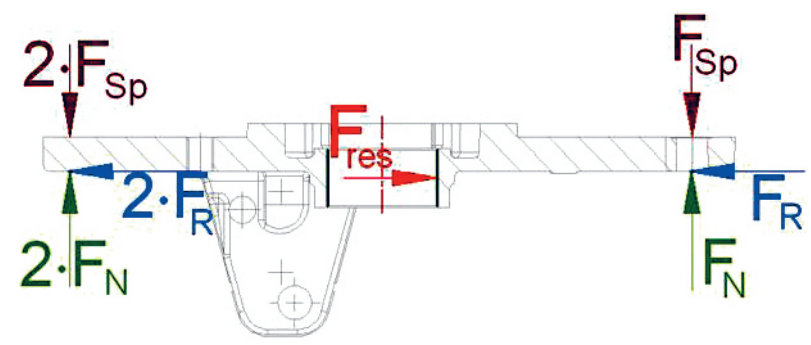

$\overline{F_{x}}=0: 3 \cdot \mu_{0} \cdot F_{s p}-x \cdot F_{r e s}=0$

$$
F_{s p}=\frac{X \cdot F_{r e s}}{3 \cdot \mu_{0}}
$$

Abb. 6: Kräfteplan für die Trägerplatte in der 1. Aufspannung Legende: $F_{x}=$ Summe der Kräfte in $X$-Richtung; $F_{\text {res }}=$ Resultierende Schnittkraft; $F_{\mathrm{R}}=$ Reibungskraft; $F_{\mathrm{N}}=$ Normalkraft; $F_{\text {sp }}=$ Spannkraft; $x=$ Korrekturfaktor für Schnittkraft; $\mu_{0}=$ Haftreibungszahl

Spannsysteme $\mathrm{GmbH}$ ) liefert hierzu in den wichtigsten CAD-Formaten wie CATIA, SolidWorks, Inventor, SolidEdge und den wichtigsten neutralen Formaten STEP, IGES, SAT, die 3D-Daten der Vorrichtungselemente.

Zur effizienten Verwendung des Vorrichtungsbaukastens der Firma Hohenstein Vorrichtungsbau und Spannsysteme $\mathrm{GmbH}$ an der TH Wildau wurden durch eine Studentengruppe alle Vorrichtungsteile des Baukastens in eine Featureverwaltung (Bibliotheksfeatures) eingepflegt. Damit ist es möglich, übersichtlich und komfortabel im 3D-CAD-System SolidWorks eine Baukastenvorrichtung zusammenzubauen. Die vollständig erstellte Baukastenvorrichtung der Trägerplatte für die 1. Aufspannung wird in Abb. 7 a) dargestellt. Um den Ablauf zu verdeutlichen, ist dazu ist die real montierte Vorrichtung für die Trägerplatte in Abb. 7 b) gegenübergestellt.

Für die Montage der Vorrichtung wird neben einem Montageplan eine Stückliste benötigt. Die zu verwendenden Einzelteile werden m. H. der Stückliste aus der Werkzeugausgabe entnommen (Lagerverwaltungssoftware). Der Montageplan gibt dem Vorrichtungsmonteur vor allem die Reihenfolge der zu montierenden Einzelteile, Baugruppen und Unterbaugruppen vor. Beispielhaft wird der Montageplan für die Unterbaugruppe Schwertbolzen in Abb. 8 dargestellt.

\section{Schlussfolgerung und Ausblick}

Das im Studiengang Ingenieurwesen/Maschinenbau investierte Baukastensystem der Firma Hohenstein Vorrichtungsbau und Spannsysteme GmbH wurde im a)

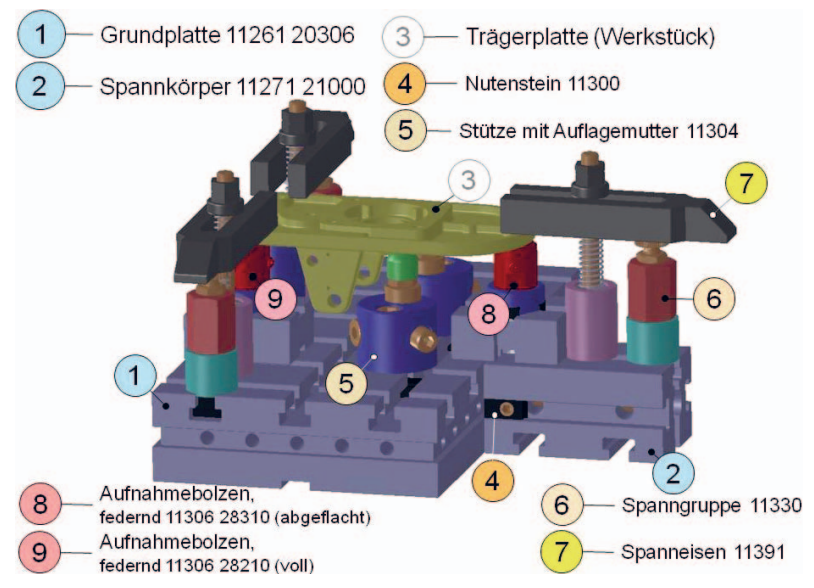

b)

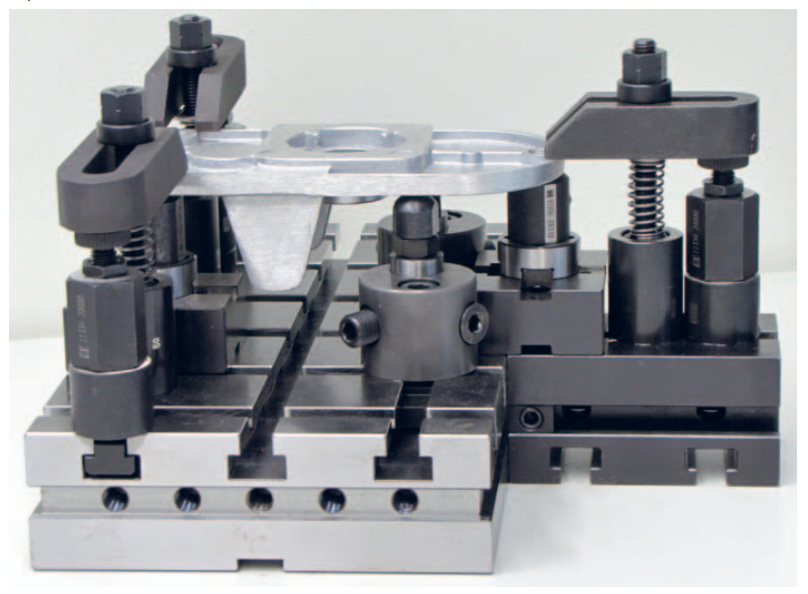

Abb. 7: Baukastenvorrichtung für das Werkstück »Trägerplatte« in der 1. Aufspannung; a) Vorrichtung im 3D-CAD-System "SolidWorks« virtuell montiert; b) Vorrichtung mit »Trägerplatte« für die Fertigung real montiert

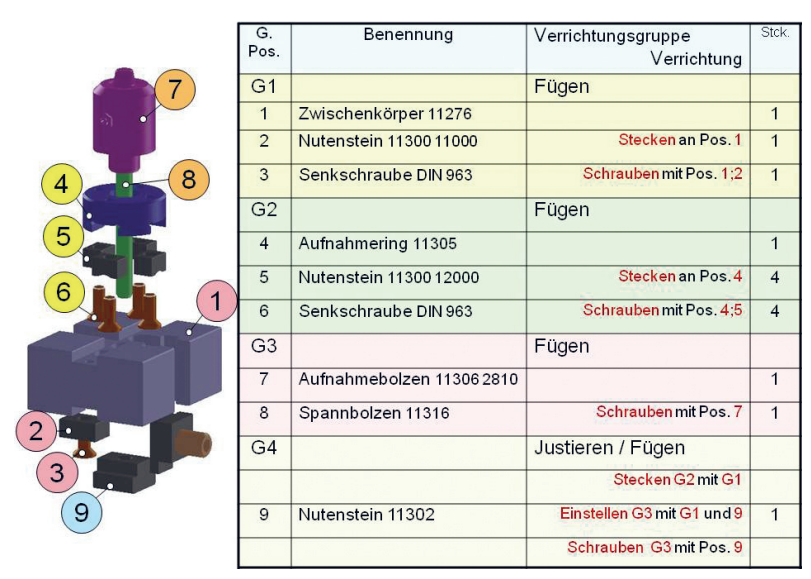

Abb. 8: Montageunterweisung für die Unterbaugruppe »Schwertbolzen«

Lehrgebiet Vorrichtungskonstruktion erfolgreich eingeführt. In Zukunft wird der Baukasten in Lehre, Technologietransfer und Forschung genutzt.

Durch die Aufbereitung der Vorrichtungsproblematik für die Lehre konnte die Vorgehensweise bei der 
Nutzung von Baukastenvorrichtungen gegenüber Sondervorrichtungen herausgearbeitet werden. Ein repräsentatives Beispiel stellt den Ablauf beispielhaft dar.

Der vorliegende Artikel klärt zunächst Fachbegriffe zum Vorrichtungsbau. Die Problematik wird auf die mechanische Bearbeitung eingegrenzt. Danach werden Sonder- und Baukastenvorrichtungen gegenübergestellt und bewertet.

Im Weiteren wurde der Planungsablauf, anhand konkreter Daten am Beispiel einer »Trägerplatte« beispielhaft dargestellt.

Es stellte sich heraus, dass die Vorrichtungsproblematik sehr komplex ist. In der Vorrichtungsplanung und -konstruktion sind technologische und konstruktive Kenntnisse erforderlich. Der im Artikel beschriebene Ablauf hebt diese Komplexität im Besonderen hervor.

Zukünftig können mit dem vorhandenen Baukastensystem auch konkrete Aufgabenstellungen aus der Industrie bearbeitet werden. In der Abarbeitung der Aufgabenstellungen ist eine interdisziplinäre Zusammenarbeit in den Laboren des Fachbereiches I/WI an der TH Wildau möglich.

Für das repräsentative Beispiel Trägerplatte sind noch folgende Teilaufgaben abzuarbeiten (siehe auch Einhaltung des Ablaufes im Abb. 1b):

1. Vermessung der montierten Vorrichtung (Lage der

Bestimmelemente).

2. Erstellung des NC-Programmes für die Bearbeitung.

3. Bearbeitung der Trägerplatte.

4. Kontrolle (Vermessung) der Trägerplatte.

5. Baukastenvorrichtung für die 2. Aufspannung.

Zusammenfassend kann festgestellt werden, dass die Anschaffung des Baukastensystems im Zusammenhang mit der komplexen und interdisziplinären Abarbeitung der Aufgabenstellung unter Nutzung modernster Hardund Software den Studenten eine auf hohem Niveau fundierte Ausbildung bietet, die auch eine Integration von Industrieprojekten möglich macht.

\section{Kurzzeichenverzeichnis}

\begin{tabular}{|c|c|c|}
\hline Kurzzeichen & Beschreibung & Einheit \\
\hline A-Plan & Arbeitsplan & \\
\hline VK-Konzept & Vorrichtungskonzept & \\
\hline NC-Prog & NC-Programmierung & \\
\hline SiMu & NC-Simulation & \\
\hline $\mathrm{BAZ}$ & Bearbeitungszentrum & \\
\hline $\mathrm{N}$ & Drehzahl & $\min ^{-1}$ \\
\hline $\mathrm{F}_{\mathrm{sp}}$ & Spannkraft & $\mathrm{N}$ \\
\hline $\mathrm{F}_{\mathrm{H}}$ & Handkraft & $\mathrm{N}$ \\
\hline I/WI & $\begin{array}{l}\text { Ingenieurwesen/Wirtschafts- } \\
\text { ingenieurwesen }\end{array}$ & \\
\hline$v_{f}$ & Vorschubgeschwindigkeit & $\mathrm{mm} / \mathrm{min}$ \\
\hline$v_{c}$ & Schnittgeschwindigkeit & $\mathrm{m} / \mathrm{min}$ \\
\hline$T_{v}$ & Vorrichtungstoleranz & $\mu \mathrm{m}$ \\
\hline $\mathrm{T}_{\mathrm{w}}$ & Werkstücktoleranz & $\mu \mathrm{m}$ \\
\hline $\mathrm{L}_{\mathrm{vB}}$ & Länge des vollen Bolzens & $\mathrm{mm}$ \\
\hline $\mathrm{L}_{\mathrm{SB}}$ & Länge des Schwertbolzens & $\mathrm{mm}$ \\
\hline $\mathrm{F}_{\mathrm{x}}$ & Summe der Kräfte in X-Richtung & $\mathrm{N}$ \\
\hline $\mathrm{F}_{\text {res }}$ & Resultierende Schnittkraft & $\mathrm{N}$ \\
\hline$F_{R}$ & Reibungskraft & $\mathrm{N}$ \\
\hline $\mathrm{F}_{\mathrm{N}}$ & Normalkraft & $\mathrm{N}$ \\
\hline$x$ & Korrekturfaktor für Schnittkraft & \\
\hline KMG & Koordinatenmesgerät & \\
\hline$\mu_{0}$ & Haftreibungszahl & \\
\hline
\end{tabular}

\section{Anmerkung}

[1] Einem Werkstück können maximal sechs Freiheitsgrade entzogen werden. Es sind drei Dreh- und drei Verschiebungsfreiheitsgrade. Der entzogene Freiheitsgrad wird i.d.R. im Vorrichtungsbau in einer Richtung entzogen. Für die andere Richtung ist dabei konstruktiv ein Spann- oder Hilfsspannelement vorzusehen. 
Literatur

Conrad, K.-J. (2010): Grundlagen der Konstruktionslehre, Methoden und Beispiele für den Maschinenbau, 5 . Auflage, Carl Hanser Verlag, München.

Dolmetsch, H., Kilgus, R., Holznagel, D., Klein, W., Keller, E., Odenwald, T. (2007): Metalltechnik Fachbildung, Der Werkzeugbau, 14. Auflage, Verlag Europa Lehrmittel, Nourney, Vollmer GmbH \& Co. KG.

Matuszewski, H. (1986): Handbuch Vorrichtungen, Konstruktion und Einsatz, Friedr. Vieweg \& Sohn Verlag/GWV Fachverlage GmbH, Wiesbaden.

Miersch, N., Busse,G., Fricke, J., Stengl, A. (2003): Analyse und Auswertung von Besonderheiten und Problemen bei der Konstruktion einer Serienvorrichtung im Rahmen einer praxisnahen Aufgabenstellung für die Lehre, Wissenschaftliche Beiträge der TFH Wildau, Heft 1 / 2003, 52 - 57

Perovic, B. (1999): Werkzeugmaschinen und Vorrichtungen, Berechnung und Konstruktion, Carl Hanser Verlag.

Trummler, A., Wiebach, H. (1994): Vorrichtungen der Produktionstechnik, Friedr. Vieweg \& Sohn Verlag/GWV Fachverlage GmbH, Wiesbaden.

VDI-Gesellschaft Produktionstechnik (1992): Vorrichtungen, Rationelle Planung und Konstruktion, VDI-Verlag GmbH, Düsseldorf.
Autor

Prof. Dr.-Ing. Norbert Miersch

Maschinenbau/Werkzeugkonstruktion

Technische Hochschule Wildau [FH]

norbert.miersch@th-wildau.de 